\title{
Le miracle éducatif asiatique existe-t-il ?
}

Réflexions autour d'un colloque international sur l'éducation en Asie Does the Asian education miracle really exist? Reflections on an international conference on education in Asia ¿Existe el milagro educativo asiático? Reflexiones acerca de un coloquio internacional sobre la educación en Asia

\section{Nathalie Mons}

\section{OpenEdition} Journals

Édition électronique

URL : https://journals.openedition.org/ries/4376

DOI : $10.4000 /$ ries. 4376

ISSN : 2261-4265

Éditeur

France Education international

Édition imprimée

Date de publication : 4 avril 2015

Pagination : 151-156

ISBN : 9782854206067

ISSN : $1254-4590$

\section{Référence électronique}

Nathalie Mons, "Le miracle éducatif asiatique existe-t-il ? », Revue internationale d'éducation de Sèvres [En ligne], 68 | avril 2015, mis en ligne le 04 avril 2017, consulté le 03 juillet 2021. URL : http:// journals.openedition.org/ries/4376 ; DOI : https://doi.org/10.4000/ries.4376 


\title{
Le miracle éducatif asiatique existe-t-il ?
}

\section{Réflexions autour d'un colloque international sur l'éducation en Asie}

\author{
Nathalie Mons \\ Conseil national d'évaluation \\ du système scolaire, \\ France
}

Il me revient de proposer une synthèse des journées passées ensemble à l'occasion de ce colloque international consacré à l'éducation en Asie ${ }^{1}$. Je voudrais commencer par une sensation : celle d'un voyage qui a duré plusieurs jours. Cela est dû évidemment à la richesse de ces journées puisqu'il y avait 24 études de cas exposées, 45 intervenants venus de 18 pays et d'organisations internationales, donc de nombreuses rencontres humaines et beaucoup de matériaux délivrés - notamment un nombre considérable de tableaux statistiques. Mais - et c'est tout l'intérêt de ces journées - il y a eu aussi des témoignages concrets, des présentations de corpus de chercheurs avec des entretiens, bref tout un ensemble qui a finalement constitué une richesse extrêmement importante. De plus, dans certains ateliers, des intervenants ont pu montrer, à travers leurs trajectoires de vie, la complexité des systèmes éducatifs qu'ils avaient fréquentés, des influences qu'ils avaient subies - coloniale, postcoloniale, soviétique, chinoise, etc. -, et la façon dont elles se sont mêlées au présent. On pouvait aussi consulter la documentation préparée par le centre de ressources et d'ingénierie documentaires du CIEP, avec une bibliographie riche de plus de 600 références et tout un ensemble de statistiques et de fiches pays ${ }^{2}$. Cette richesse et cette variété expliquent ma sensation d'un voyage qui a duré dans le temps et la difficulté, par conséquent, de synthétiser ces échanges.

Cette synthèse ne prétend évidemment pas à l'exhaustivité - ce serait tout à la fois immodeste et impossible. Je prétends au contraire à la trahison puisque je n'ai pu assister à tous les ateliers : je rapporte, en les déformant, des éléments que j'ai entendus moi-même ou qui m’ont été transmis, et je ne proposerai qu'une généralisation hasardeuse puisque, au fil du colloque, c'est bien la

1. Cet article est la transcription révisée d'une conférence de synthèse proposée par l'auteur le 14 juin 2014 , lors du colloque international « L'éducation en Asie en 2014 : quels enjeux mondiaux ? », organisé du 12 au 14 juin 2014 par le Centre international d'études pédagogiques (CIEP), à l'occasion du $20^{\mathrm{e}}$ anniversaire de la Revue internationale d'éducation de Sèvres. Le programme de ces journées et les communications mises en ligne sont consultables sur : http://ries.revues.org/3710/(NdIR)

2. Ces documents sont consultables en ligne sur le site du CIEP : http://goo.gl/gjKvyz/. Les statistiques et les fiches pays concernent les pays ou territoires suivants : Cambodge, Chine, Corée du Sud, Hong-Kong, Inde, Indonésie, Japon, Malaisie, Pakistan, Singapour, Sri Lanka, Taïwan, Thaïlande, Vietnam. (NdIR) 
variété des expériences, des cas nationaux, des contextes, qui a été mise en évidence. De plus, certains concepts n'étaient pas stabilisés, puisqu'il a été question de cultures, de civilisations, des notions très complexes que l'on n'a pu développer dans ce cadre.

Finalement, pourquoi étions-nous là ? C'est la première question que je me pose et que nombreux se sont posée, durant le colloque. Pourquoi avons-nous eu la chance de cette très belle rencontre ? Différents intervenants sont revenus sur le titre du colloque : «L'éducation en Asie en 2014 : quels enjeux mondiaux? ». Certains ont souligné que l'Asie était au singulier dans le titre mais au pluriel dans la note de cadrage ${ }^{3}$ qui accompagnait le programme. Donc pourquoi étionsnous là ? Il est certainement intéressant d'ausculter les différentes dimensions possibles de la réponse, car elles peuvent nous donner les clés de ce que nous sommes venus chercher dans ces rencontres.

Il est évident que la première démarche est celle de la découverte : découverte d'un continent, d'expériences fascinantes dont on a entendu parler. Il s'agit bien d'une curiosité intellectuelle nourrie de la conviction que les rencontres sont irremplaçables. Pour avoir vu les interactions qui se sont nouées durant les pauses, ces espaces de liberté, on ne pouvait qu'être sensible à la fascination pour la rencontre humaine et pour la découverte en général, qui s'est manifestée.

Mais cela n'est pas tout, et le titre du colloque le dit bien. "L'éducation en Asie pour 2014 : quels enjeux mondiaux? " n'invite pas seulement à une description. Il propose de partir à la découverte d'expériences nationales étrangères, parce qu'elles nous renvoient à une réflexion sur nos propres destinées, sur nos propres situations, sur nos propres difficultés. On y lit la fascination pour ce miroir asiatique qui renvoie les participants venus d'autres pays du monde à des analyses nationales voire personnelles extrêmement complexes. La notion de "réussite asiatique " évoque évidemment les questions d'éducation dans un tel colloque, mais avec la conscience de l'arrière-plan économique et du dynamisme démographique qui interrogent aussi l'Occident. Le miroir que nous tend l'Asie est en outre celui des réformes, au moment où, à l'Ouest, les systèmes peuvent apparaître comme quelque peu bloqués. Or on voit en Asie du mouvement, une dynamique, si bien que le miroir asiatique fascine et invite à une forme d'introspection.

Cette fascination mêlée de représentations imaginaires confine parfois - cela a été évoqué dans les ateliers - à la caricature. C'est tout l'intérêt d'un tel colloque que de tenter de dépasser le stade des généralisations abusives, notamment au sujet de ces pays géants que sont l'Inde et la Chine. Par exemple, on est revenu dans plusieurs ateliers sur le rote learning ou apprentissage par répétition : est-ce vraiment à cela que se résume la pédagogie en Asie ? De quoi 
s'agit-il en réalité ? Pour prendre un autre exemple, cette image des pays asiatiques en tête de PISA n'est-elle pas encore une caricature? Quels sont finalement ces États-nations et représentent-ils l'Asie? Les participants au colloque ont travaillé collectivement pour tenter de dépasser quelques stéréotypes.

Enfin, certains termes sont revenus régulièrement dans les ateliers, qu'il est important de mentionner : «dangers », " risques », " paix », "guerre »... Fascination et peur ont souvent partie liée, en particulier dans les relations avec l'étranger. Du côté Ouest, peur du déclassement. «Qui classe, déclasse » a déclaré un participant, et cet aspect explique aussi pourquoi nous participions à ce colloque. Il faut se rappeler que PISA structure aujourd'hui largement, grâce à la force de communication de l'OCDE, les dialogues en éducation et les comparaisons internationales. Si l'on revient sur la création historique de ces outils, qu'il s'agisse de PISA ou de ses prédécesseurs, on relève que les premiers outils de comparaison internationale des acquis des élèves furent lancés dans les années 1960 dans un contexte de guerre froide, suite au lancement par les Soviétiques du satellite Spoutnik. Donc il y a toujours eu cette tension sous-jacente, dans les comparaisons internationales, d'une part entre la curiosité intellectuelle, la découverte, la volonté de savoir ce qui se passe ailleurs et de nourrir la réflexion de la connaissance des pratiques et des politiques éducatives innovantes et, d'autre part, une inscription de ces comparaisons dans un contexte de compétition internationale entre pays. Cet aspect géopolitique reste évidemment à l'arrière-plan de nos réflexions actuelles.

Enfin, je crois qu'il est important de remettre en perspective la fascination - qu'on peut aussi qualifier de perplexité, voire de défiance - de l'Occident pour l'Orient : elle n'est pas nouvelle et relève d'une longue histoire sur plusieurs plans. Il faut en être conscient pour être en mesure d'analyser les échanges produits lors de ce colloque.

Enfin, si nous savons qu'en éducation non plus, la comparaison internationale n'est pas nouvelle, on peut observer cependant que souvent, elle se développe dans des contextes de crise, lors des fractures, des grands changements. Donc la comparaison n'est nouvelle ni dans le domaine scientifique ni dans celui des politiques publiques, même si elle emprunte différentes voies. Il me semble que ce colloque a réussi à éviter deux écueils manifestes, en quelque sorte deux voies extrêmes, grâce à la richesse du programme proposé.

La première de ces deux voies extrêmes est celle du culturalisme, qui considère chaque expérience, souvent nationale, comme singulière, rendant impossible toute forme de comparaison, car l'école est façonnée par la société. Mark Bray a rappelé en ouverture la nécessité de distinguer des unités de comparaison et d'appréhender les différents contextes non seulement au niveau national mais aussi à des niveaux infranationaux ${ }^{4}$, et $\mathrm{j}^{\prime}$ ajouterais infrarégionaux, en ce 
qui concerne l'Asie. Par nature, toute comparaison comporte la difficulté de trouver une unité de comparaison et exige de vérifier si la singularité des niveaux de comparaison choisis empêche ou non la comparaison.

L'autre voie extrême est celle de la recherche de modèles, qui seraient « hors sol ». C'est évidemment ce qui se produit de façon récurrente avec PISA : on regarde les résultats des pays asiatiques et on se demande pourquoi ils réussissent, comme s'il était possible de définir un modèle asiatique d'éducation ${ }^{5}$.

S’il me semble que ces journées sont réussies, c'est parce que nous avons pu collectivement trouver une voie moyenne entre le refus de la comparaison lié à la singularité de chacun des contextes et le refus de la généralisation abusive de modèles hors sol. Les interactions au sein des ateliers ont très souvent porté sur cette recherche d'équilibre.

Alors qu'avons-nous découvert, finalement, après cette journée et demie ? Trois points guident ma réflexion.

Nous avons, me semble-t-il, d'abord découvert que le miracle asiatique n'existe pas en éducation. Je vais développer ce point. De nombreuses questions finalement non résolues dans les différents pays ont été mises en avant, et tout cela crée des points communs non seulement du côté asiatique, mais aussi avec les autres pays - c'est un aspect intéressant, qui permet d'établir le dialogue. Si l'on peut dire, de façon assez rapide, que l'Asie et le miracle asiatique n'existent pas en éducation, les ateliers ont montré qu'il existe des singularités de certaines Asies. Enfin, en tant que sociologue, je voudrais rappeler qu'il est souvent intéressant de travailler, notamment en ce qui concerne les politiques publiques, sur ce que font les acteurs et ce qu'ils ne font pas. Le vide nous intéresse et nous interpelle toujours autant que le plein.

Si je disais que l'Asie et le miracle asiatique en éducation n'existent pas, de façon un peu provocante, c'est pour souligner qu'il existe bien des Asies et non une Asie. L'afflux de statistiques présentées lors de la première journée du colloque le montrait amplement : pour l'Asie du Sud et du Sud-Est, on observe des décalages très importants en ce qui concerne l'économie; il existe une dynamique générale mais qui n’a pas la même intensité selon les régions. En matière de transition démographique, les différents espaces asiatiques en sont également à des périodes de maturité différente, et tout ceci, évidemment, questionne les systèmes éducatifs de façon totalement différente. Par ailleurs, les inégalités se développent mais pas partout de la même façon, la pauvreté existe toujours dans un certain nombre de pays asiatiques, et ces éléments interrogent l'imaginaire que j'évoquais plus haut autour d'une croissance asiatique.

Deuxièmement, en ce qui concerne l'éducation, cet imaginaire du miracle asiatique n'existe pas non plus. Dans plusieurs ateliers, les résultats au PISA ont été examinés et on s'est interrogé sur les territoires censés représenter

5. Cette question était au cœur de l'atelier 6. Voir dans ce numéro 68 l'article de Jean-Marie De Ketele et Bernard Hugonnier (2015) : « Peut-on parler de modèle(s) asiatique(s) et quels enseignements en tirer ? », p. 131-140 (NdIR) 
l'Asie : que représente le cas de Shanghai à l'échelle du pays, ou une cité-État telle que Singapour, par exemple? Au cours du colloque, la forte présence asiatique dans les meilleurs scores de PISA a été soulignée d'un côté, mais de l'autre, on a vu que la problématique de l'éducation pour tous était au cœur des discours et des interrogations dans certains pays.

Un autre miracle asiatique a été mis en perspective dans l'atelier consacré au financement de l'éducation, ainsi que dans celui consacré à l'éducation de l'ombre et au tutorat privé : celui de coûts de l'éducation faussement faibles. De même, on a constaté qu'en Asie aussi s'expriment de fortes interrogations, par exemple au sujet de l'apprentissage par résolution de problème ou par répétition.

Enfin, un dernier élément nous invite à réfuter l'idée d'un miracle éducatif asiatique : l'existence même de questions multiples, que l'on trouve aussi bien en Asie qu'en Europe, en Afrique et dans les Amériques. Il s'agit de savoir comment fabriquer de l'éducation dans des contextes nationaux. Dans l'atelier consacré aux curricula, les interrogations ont porté notamment sur les valeurs centrales, nationales, que les contenus d'enseignement doivent refléter. Quelles influences internationales sont au cœur des réflexions? La place des examens, des pressions scolaires a été soulignée dans plusieurs ateliers, tout comme a été questionné ce que doit être le curriculum commun à tous les élèves : quand faut-il proposer des formes de différenciation? Doit-on créer des écoles spécifiques pour certains publics? Quid de l'obsolescence et de l'innovation pédagogiques, à partir des cas très intéressants de Singapour, de Hong-Kong, etc. ? Ces questions se posent aussi bien à l'Est qu'à l'Ouest. De même, en ce qui concerne la place du marché et du privé, et ce qu'elle dit de la confiance ou de la défiance vis-à-vis de l'institution publique. Il semble qu'en Asie aussi, l'on en soit aujourd'hui à la croisée des modèles, à l'absence de modèle unique. Nous vivons dans des sociétés en transition, dans lesquelles se croisent et vont se croiser des modèles de société différents. Sans compter qu'en Asie aussi, la culture juvénile, qui passe par Internet, remet en cause les cultures scolaire et familiale. De nombreux intervenants ont rappelé la superposition, en Asie, des modèles coloniaux, postcoloniaux, néocoloniaux et leurs relations avec les cultures traditionnelles, puis l'apparition d'une nouvelle culture, dans un contexte de mondialisation. Donc la complexité existe partout sur la planète et la question partout posée est bien de savoir comment en tirer parti.

S'il est donc impossible de nourrir le fantasme d'un miracle asiatique en éducation, ce constat n'exclut pas qu'on ne puisse se nourrir des réflexions des Asies, comme l'ont montré ces journées fécondes en analyses de cas et en démonstrations de problèmes. C'est en entamant les réflexions pédagogiques de façon pratique, à partir d'analyses de cas spécifiques, que l'on a pu mettre en perspective les réformes holistiques qui se sont produites dans de nombreux pays d'Asie, où l'on observe la volonté de penser de façon globale les réformes et non, 
comme dans nos vieux pays, notamment européens, de façon plutôt segmentée. C'est certainement cette vision holistique globale qui entraîne la capacité de changement, à quoi il faut ajouter la coexistence de modèles multiples au sein de sociétés en transition perpétuelle, peut-être davantage qu'en Europe notamment.

Certes, certains pays ou territoires asiatiques sont actuellement ceux qui réussissent le mieux dans les comparaisons internationales en éducation. Mais il aurait pu être intéressant, au cours de ces trois journées, de relever également qu'on observe, depuis dix ans, un accroissement des inégalités scolaires d'origine sociale à l'école dans ces pays. En d'autres termes, il aurait aussi été intéressant de questionner PISA et le "soft power». Pourquoi la Chine est-elle représentée par Shanghai dans PISA ? Les instruments internationaux servent aussi des diplomaties, notamment culturelles et de soft power. En la matière, l'habileté n'est pas moindre en Orient qu'en Occident.

Enfin ce colloque aura également fait émerger une autre question, qui mérite d'être travaillée, et qui est celle des relations intra-Asie. Dans ces Asies extrêmement dynamiques qui ont émergé, les classes moyennes, désormais majoritaires, auront-elles encore besoin des autres parties du monde ou se suffirontelles à elles-mêmes? 